\title{
THERAPEUTIC RESPONSE OF WILSON'S DISEASE TO D-PENICILLAMINE IN PAEDIATRIC POPULATION: A ONE YEAR FOLLOW-UP STUDY
}

\author{
SAHA NC ${ }^{1}$, SULTANA A ${ }^{2}$, MOLLAH MAH ${ }^{3}$, BEGUM T $^{4}$, RAHMAN AKMM ${ }^{5}$, FATMI LE 6
}

\begin{abstract}
:
Objectives: The objective of this study was to observe the outcome of patients treated with penicillamine.

Design: Intervention type of study

Setting: Department of Paediatrics, Dhaka Medical College Hospital

Study period: January 2007 to December 2008.

Study subjects: Sixteen diagnosed cases of Wilson's disease as per inclusion criteria.

Intervention: D-penicillamine was started in a low dose, which was titrated gradually. The clinical and biochemical parameters were evaluated to look for the response to treatment.

Results: A total of 16 cases were included. Among them 12 were male and 4 were female. The mean $( \pm S D)$ of age of the patients was 10 ( \pm 2.34$)$ years. Consanguinity between parents was present in $44 \%(n=7)$. The hepatic and neurological variety of WD were $56 \%(n=9)$ and $44 \%$ $(n=7)$ respectively. The K-F ring was present in $75 \%(n=12 / 16)$ of WD cases. The excretion of $24 \mathrm{hrs}$ urinary copper was steadily increased from discharge till second follow-up in response with increasing dose of penicillamine, thereafter the value was declining gradually till final follow-up at 1 year. Regarding outcome, 7 patients improved of which 4 were in hepatic and 3 in neurological group, 3 of hepatic WD expired and 2 developed neurological manifestations. One patients developed proteinuria while penicillamine treatment. About half of patients with WD were improved. Adequate cupriuresis occurred at three months. All the symptoms and biochemical markers WD improved gradually. No significant side effect was seen.
\end{abstract}

Key words: Wilson's disease, penicillamine, urinary copper.

J Dhaka Med Coll. 2009; 18(1) : 37-43

\section{Introduction:}

Wilson's disease (WD) is a rare autosomal recessive disorder with a prevalence of 1 in 30,000 to 1 in Wilson's disease (WD) is a rare autosomal recessive disorder with a prevalence of 1 in $100000^{1}$. It is characterized by inability of the liver to transport and store normally absorbed dietary copper, resulting in abnormal deposition of copper occurs in the liver, basal ganglia, eyes, and other tissues ${ }^{2}$.

Treatment modalities include avoidance of copper containing diet; pharmacotherapy with copper chelating agents; of these D-
Penicillamine is the oldest and most commonly used drug, which is recommended till today for the initial management of symptomatic $\mathrm{WD}^{3-7}$. Though few reports on use of zinc as treatment modality have been described but there is no universal agreement regarding its use as a single agent. Penicillamine promotes the urinary excretion of copper. The use of lower initial doses, increasing over few weeks, can increase tolerance to the drug ${ }^{1}$. Adequacy of treatment can be monitored by measuring $24-$ hour urinary copper excretion while on treatment ${ }^{1,5}$. Response to treatment with D-

1. Associate Professor, Division of Child Neurology, Department of Pediatrics, Dhaka Medical College, Dhaka

2. FCPS Student, Department of Pediatrics, BSMMU, Dhaka

3. Professor, Department of Pediatrics, Dhaka Medical College, Dhaka

4. Professor, Department of Pediatrics, Ibrahim Medical College \& BIRDEM Hospital, Dhaka

5. Assistant Professor, Department of Pediatrics, Dhaka Medical College, Dhaka

6. Professor, Department of Pediatrics, Dhaka Medical College, Dhaka.

Correspondence: Dr. Narayan Chandra Saha. 
penicillamine has been variously described. Following treatment with penicillamine, the 24 hrs excretion of copper increases gradually at the initial phase, thereafter it begins to fall again with exhaustion of abnormally deposited copper ${ }^{4,5}$. Though we are using penicillamine, not enough data is available in our population regarding response to penicillamine. The objective of this study was to document the outcome of WD treated with penicillamine with regard to clinical and biochemical parameters.

\section{Materials and Methods:}

This study was done in Dhaka Medical College Hospital from January 2007 to December 2008. All Paediatric patients of Wilson's disease admitted in study place were included who fulfilled the inclusion and exclusion criteria. Diagnosis of Wilson's disease was based on compatible clinical feature as described below plus investigation profiles. Criteria for inclusion of different types of Wilson's disease in pediatric population ( $<15 \mathrm{yrs}$ ) were as follows $1,3,4,8,9$

1) Neurological Wilson's disease: was defined if patient had history of:

a. Regression of acquired milestones-like progressive deterioration of school performance and of handwriting or deterioration of speech and motor function.

b. Features of Basal ganglia lesion in the form of choreo-athetosis or dystonia with positive laboratory findings: Serum ceruloplasmin $<20 \mathrm{mg} / \mathrm{dl}, 24 \mathrm{hrs}$ urinary copper (UC) > 100 $\mathrm{gg} /$ $24 \mathrm{hrs}$, D-Penicillamine challenge test: $24 \mathrm{hrs}$ urinary copper $>1200 \mu \mathrm{g} /$ day, presence of K-F ring on slit lamp examination.

2) Hepatic Wilson's disease: patient with hepatitis not explainable to common causes but who fulfills compatible laboratory findings as mentioned above.

A final diagnosis was made if a patient fulfilled at least two of the following criteria ${ }^{1-4}$ in addition to compatible clinical feature i.Presence of $\mathrm{K}-\mathrm{F}$ ring on slit lamp examination ii. Low serum ceruloplasmin level $(<20 \mathrm{mg} / \mathrm{dl})$ iii. High urinary copper excretion $(>100 \mu \mathrm{g} / 24$ hrs) iv. D-Penicillamine challenge test (UC $>1200 \mu \mathrm{g} / 24 \mathrm{hrs}$ )
Exclusion criteria:

Progressive neurological disease, hepatic or other clinical features compatible with WD but laboratory parameters are negative.

\section{Study procedure:}

Patients who were found eligible with regards to inclusion and exclusion criteria were enrolled after taking consent from parents. Data was collected by pre-designed structured questionnaire. After enrolment, detailed history was taken; systemic examinations and relevant laboratory investigations were done. The clinical profile and laboratory results were evaluated by a trained paediatric neurologist. Urinary copper excretion was done in all patients by "Atomic energy spectrophotometry" (normal range $<40 \mu \mathrm{g} / 24 \mathrm{hrs}$ ) from Atomic energy centre laboratory, Dhaka. Serum ceruloplasmin level was estimated by "Radial Immunodiffusion method" RN045.3 Bindarid UK, from AFIP.

Liver function tests and serologic markers for common hepatotrophic viruses like HAV, HEV, $\mathrm{HBV}$, and $\mathrm{HCV}$ were also done to exclude other liver diseases. Routine tests like $\mathrm{CBC}$, urine $\mathrm{R} / \mathrm{E}$ (to detect proteinuria), serum creatinine was also done to monitor toxic effects of drugs in addition to clinically documented side effects.

Once a final diagnosis was achieved, the child was treated with D-Penicillamine (Cap Artamine $250 \mathrm{mg}$, Chandra Bhagat Pharma, Mumbai) at a dose of $5 \mathrm{mg} / \mathrm{kg} /$ day then titrated with an increment of $5 \mathrm{mg} / \mathrm{Kg}$ every fortnightly to a maximum of $30 \mathrm{mg} / \mathrm{kg} /$ day $^{10}$. In Hepatic WD increase in the dose up to $20 \mathrm{mg} / \mathrm{kg} /$ day. Drugs were given before food in divided doses (after crushing granules of the capsule) along with supplementation of low copper diet and pyridoxine. Cases were then followed up for clinical and laboratory outcome by the same physician who initially assessed the cases. Neurological assessment was done for muscle power through Medical Research Council (MRC) scale $(0-5,5=$ normal power $)$, Grading of reflex $(0-$ $4+, 4+$ indicates clonus) and assessment of disability by a scoring system like Barthel Index (BI index), a score of $100 \mathrm{BI}$ is 
continent,feeds, dresses, walks, ascends and descends himself and the severity of dystonia (Level 1-4, Level 4 = massive dystonia) was also assessed ${ }^{10-12}$. Patients were monitored three monthly by estimating urinary copper till 1 year follow-up period to document adequate $(24 \mathrm{hrs}$ Urinary copper $2-5 \mathrm{mg} /$ day) and maintenance cupriuresis (24 hours Urinary copper excretion 0.5-1 mg/day) along with clinical parameter ${ }^{5}$. The time needed for adequate and maintenance cupriuresis was recorded. The dose and duration of drug therapy was also recorded. After completion of 1-year follow up study, patients were advised to continue the drug and to come for follow up.

\section{Statistical analysis:}

All clinical and biochemical parameters were recorded before and after the intervention. Data was edited meticulously and entered into a computer. Data was analyzed by computerbased statistical program SPSS (Statistical Package for Social Science) for Window (version 12). Results were expressed as frequency, percentage, and mean \pm SD. For statistical analysis paired 't'test was used for comparing means of quantitative data and Chi-square test were used for qualitative data. Differences were considered statistically significant if $\mathrm{p}<0.05$.

\section{Results:}

The study was intended to observe the outcome of patients with Wilson disease following Dpenicillamine treatment. The study also intended to assess the excretion of copper through the urine at different phases of treatment. A total of 16 patients were enrolled for analysis. The mean $( \pm \mathrm{SD})$ age of the patients was $10( \pm 2.34)$ years with a range of 6-14 years. The mean $( \pm S D)$ age of the hepatic and neurological WD was 7.8 yrs and 10.7 yrs with a range of 7-8 yrs and 8-12 yrs respectively. The male: female ratio was 3:1.Consanguinity between parents was present in $44 \%(n=7)$. The proportion of hepatic and neurological variety of WD was $56 \%(n=9)$ : 44\% ( $=7$ ).

The K-F ring was present in $75 \%(n=12 / 16)$ of WD cases. It was present in all neurological WD $(n=7)$ and $55.55 \%(n=5 / 9)$ of hepatic WD. The excretion of $24 \mathrm{hrs}$ urinary copper was steadily increased from discharge till second follow-up in response to increasing dose of penicillamine, thereafter the value was declining gradually till final follow-up at 1 year (Table-I, II \& Fig.-1).

Table-I

Mean drug dose $(\mathrm{mg} / \mathrm{Kg})$ at discharge and different follow up

\begin{tabular}{|c|c|c|c|c|c|}
\hline \multirow[t]{2}{*}{ Disease type } & \multirow{2}{*}{$\begin{array}{c}\text { Dose atdischarge } \\
\mathrm{mg} / \mathrm{Kg}\end{array}$} & \multicolumn{4}{|c|}{ Dose at Follow up (mg/Kg) } \\
\hline & & First & Second & Third & Fourth \\
\hline Neurological WD & 9.29 & 30 & 30 & 30 & 30 \\
\hline Hepatic WD & 5.00 & 11.88 & 16 & 16 & 16 \\
\hline
\end{tabular}

Table-II

Mean drug dose $(\mathrm{mg} / \mathrm{kg})$ and mean 24-hour urinary copper in ig (24 hrs UC ig) excretion at discharge and different follow up

\begin{tabular}{|c|c|c|c|c|c|c|c|}
\hline \multirow[t]{2}{*}{ Disease type } & & \multirow{2}{*}{$\begin{array}{l}\text { Admission } \\
\mu \mathrm{g} / 24 \mathrm{hrs}\end{array}$} & \multirow{2}{*}{$\begin{array}{l}\text { Discharge } \\
\mu \mathrm{g} / 24 \mathrm{hrs}\end{array}$} & \multicolumn{4}{|c|}{ Follow upug / 24hrs } \\
\hline & & & & $1 \mathrm{st}$ & 2nd & 3rd & 4th \\
\hline \multirow[t]{2}{*}{ Neurological WD } & 24 hour UC & 287.14 & 1724.49 & 3391.0 & 3203.00 & 2243.57 & 1905.0 \\
\hline & Drug dose $(\mathrm{mg} / \mathrm{kg})$ & nil & 9.29 & 30 & 30 & 30 & 30 \\
\hline \multirow[t]{2}{*}{ Hepatic WD } & 24 hour UC & 360 & 1552.89 & 2263.0 & 2240.17 & 1317.40 & 936.60 \\
\hline & Drug dose $(\mathrm{mg} / \mathrm{kg})$ & nil & 5 & 11.8 & 16 & 16 & 16 \\
\hline
\end{tabular}




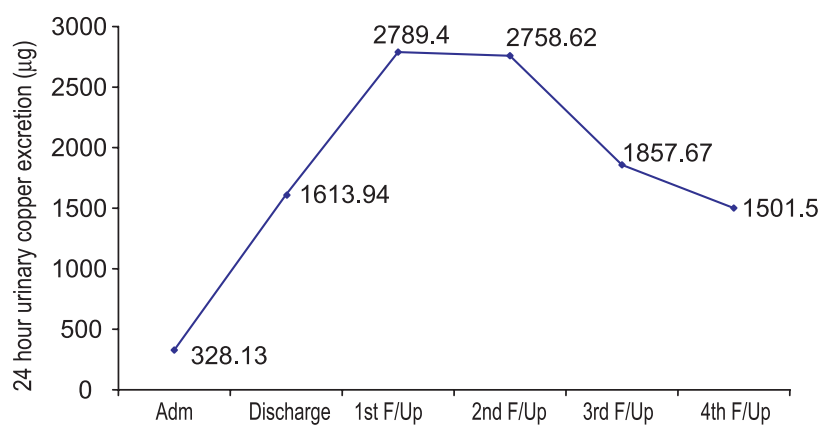

Fig.-1: Line chart showing 24-hour urinary copper excretion at admission, discharge and subsequent follow up till 1 yr.

Table-III

Shows comparison of 24 hrs urinary copper (24hrUC) at admission VS. Discharge (A), first (B), second (C), third (D) and final follow-up (E) at 1 year

\begin{tabular}{|c|c|c|c|c|c|c|c|c|}
\hline \multirow{3}{*}{$\begin{array}{l}24 \mathrm{hrs} \\
\mathrm{UC}\end{array}$} & \multicolumn{4}{|c|}{ Neurological Wilson's } & \multicolumn{4}{|c|}{ Hepatic Wilson's } \\
\hline & \multirow{2}{*}{$\begin{array}{c}\text { Mean drug } \\
\text { dose } \\
\mathrm{mg} / \mathrm{kg}\end{array}$} & \multicolumn{3}{|c|}{$24 \mathrm{hrs}$ UC } & \multirow{2}{*}{$\begin{array}{c}\text { Mean drug } \\
\text { dose } \\
\text { mg/kg }\end{array}$} & \multicolumn{3}{|c|}{24 hrs UC } \\
\hline & & $\mathrm{t}$ & $\mathrm{df}$ & $\mathrm{p}$ & & $\mathrm{t}$ & $\mathrm{df}$ & $\mathrm{p}$ \\
\hline $\bar{A}$ & 9.29 & -5.766 & 6 & .001 & 5 & -11.805 & 8 & .000 \\
\hline B & 30 & -5.451 & 6 & .002 & 11.8 & -11.882 & 7 & .000 \\
\hline $\mathrm{C}$ & 30 & -8.289 & 6 & .000 & 16 & -9.186 & 5 & .000 \\
\hline $\mathrm{D}$ & 30 & -6.894 & 6 & .000 & 16 & -10.405 & 4 & .000 \\
\hline $\mathrm{E}$ & 30 & -5.632 & 6 & .001 & 16 & -5.242 & 4 & .006 \\
\hline
\end{tabular}

Compared to admission, the 24 hrs urinary copper excretions were increased progressively in subsequent follow-up visits in response to penicillamine therapy $(\mathrm{p}<0.05)$-Table-III. At first follow up, all of neurological Wilson disease and $5(62.5 \%)$ of hepatic Wilson disease patients had adequate cupriuresis (urinary copper excretion 2-5 mg/day), none of the patient achieved a state of maintenance cupriuresis (urinary copper excretion $0.5-1 \mathrm{mg} /$ day) at $1 \mathrm{yr}$ final follow-up. The concentration of urinary copper excretion increased initially following chelation with D-Penicillamine, thereafter attained a steady state before being declined gradually in both the groups on subsequent follow up (Fig-1).

The mean value at admission of both serum bilirubin (10.14 mg/dl) and SGPT (80.56 IU/L) was decreased gradually to final value of $3.2 \mathrm{mg} /$ $\mathrm{d} 1$ and $39.60 \mathrm{IU} / \mathrm{L}$ respectively in hepatic Wilson's disease. The INR value was also decreased significantly in subsequent follow-up compared to admission $(\mathrm{p}<0.05)$. For neurological WD there was no change in the grading of muscle power but slight improvement $(p=0.05)$ in the grading of reflexes was observed, severity of dystonia and disability score (BI) showed a trend of improvement though statistically not significant till final follow-up.

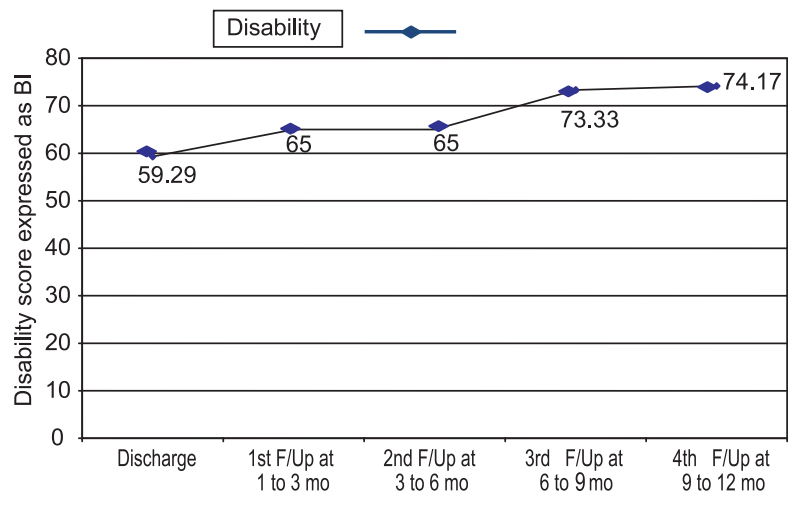

Barthel index (BI) showing improvement in disability score while on treatment

Fig.-2: Line chart showing disability score of neurological WD at admission, discharge and different follow-up. 
Table-IV

Outcome of present series ( $n=16)$

\begin{tabular}{lcccc}
\hline Type of disease & Improved & Expired & Unchanged & Developed new problem \\
\hline Hepatic WD & 4 & 3 & 0 & 2 \\
Neurological WD & 3 & 0 & 4 & 0 \\
\hline Total & 7 & 3 & 4 & 2 \\
\hline
\end{tabular}

Table 4 shows that among the hepatic WD, 4 patients improved, 3 expired and 2 developed neurological manifestations. In neurological WD, 3 patients improved and 4 cases remained unchanged.

\section{Discussion:}

The study included the patients with Wilson's disease and intended to observe the outcome of patients treated with D-Penicillamine. The mean $( \pm \mathrm{SD})$ age of the patients was $10( \pm 2.34)$ years with a range of 6-14 years. This finding is similar to the study conducted by Yuce et al $(2003)^{13}$ who found the age range from 6-15 years. The mean $( \pm \mathrm{SD})$ age of the hepatic and neurological WD was $7.8 \mathrm{yrs}$ and $10.7 \mathrm{yrs}$ with a range of 7-8 yrs and 8-12 yrs respectively. The mean age of presentation of hepatic WD at $6.8 \mathrm{yrs}$ and neurological WD at about $8 \mathrm{yrs}$ has also been reported by Kalra et al (2000) from India ${ }^{2}$.

The male female ratio of the patients was 3:1. The sex distribution is quite similar to the findings of Shakya and Agrawal (2004) who got $73.7 \%$ patients being male ${ }^{14} . .43 .75 \%$ patients have their parents with consanguineous marriage. Parental consanguinity was noted in $12.5 \%$, among hepatic WD in another study from Bangladesh ${ }^{15}$ and also from Turkey where consanguinity was as high as $70 \%$ due to intrafamily marriage which also seems to be likely in the present series ${ }^{13}$.

There is a great deal of variability in the clinical presentation, typically a hepatic WD presents in the first decade of life, neurological Wilson's generally due to extrapyramidal involvement and psychiatric symptoms usually presents later ${ }^{1,2,3}$. In broad terms, patients can present acutely with liver failure, haemolysis or both or more chronically with liver disease, neurological disease or both ${ }^{1}$. In the present study hepatic WD was more common $(56 \%, \mathrm{n}=9)$ than neurological WD $(44 \%, n=7)$ which is similar to a study from India where out of 124 paediatric patients, hepatic WD was observed in $54 \%(n=67)$ cases $^{5}$.

$\mathrm{K}-\mathrm{F}$ rings are usually present in $95 \%$ of children with neurological symptoms, $50-60 \%$ of children without neurological symptoms and in $10 \%$ of asymptomatic siblings with WD ${ }^{5} . \mathrm{K}-\mathrm{F}$ ring was present in all patients of neurological WD disease and in $55.56 \%$ patients of hepatic WD disease which corroborates with above report. Yuce et al (2003) also demonstrated the presence of $\mathrm{K}-\mathrm{F}$ ring in all patients with neurological manifestation and in 58\% patients with only hepatic presentation 13 . Shakya and Agrawal's (2004) observation also supports the present report. They reported K-F ring in $89.5 \%$ of patients ${ }^{14}$.

The mean $( \pm \mathrm{SD})$ of 24 hours urinary copper at admission was 328.13 ( \pm 169.69$)$ ig. This value is supported by Roberts and Schilsky $(2003)^{3}$. They stated that excretion of urinary copper greater that $100 \mathrm{ig} / 24$ hours is a diagnostic marker of Wilson's disease. The 24 hours urinary copper excretion with a range of 112 $2800 \mathrm{ig} / \mathrm{d}$ was also observed by Yuce et $\mathrm{al}^{13}$. After giving D-Penicillamine the mean $( \pm \mathrm{SD})$ urinary excretion of $\mathrm{Cu}$ increased to 1613.94 $( \pm 282.93) \mu \mathrm{g}$ at discharge. It was then progressively increased to a value of 2759-2790 $\mathrm{ig} / 24 \mathrm{hrs}$ at $2^{\text {nd }}$ and $3^{\text {rd }}$ follow-up, to achieve a steady state in between 3-6 months after starting penicillamine. The 24 hours excretion thereafter declined gradually to a value of $1501.50( \pm 758.06)$ ig at $1 \mathrm{yr}$-final follow-up. This observation supports the view that following treatment with penicillamine, the $24 \mathrm{hrs}$ excretion of copper increases gradually at the initial phase, thereafter it reaches a steady state until it begins to fall again with 
exhaustion of abnormally deposited copper. The majority of the patient achieved adequate cupriuresis at 3 months following penicillamine that was maintained till 6 months, thereafter started declining. None of the patient of the present study achieved maintenance cupriuresis at 1 year follow-up, documenting that more time is required to achieve that state. An improvement in the clinical features following 2-3 months of treatment, continuing over a period of 1-2 years along with an increase in 24 hours UC has also been reported in the literature ${ }^{4}$.

A marked increase in the urinary copper excretion after one week of penicillamine treatment was also described by Karim et al ${ }^{15}$. This finding is also supported by Bertrand et al (1985) 16. They stated that following DPenicillamine therapy urinary copper excretion initially increased and thereafter gradually decreased to approximately $50 \%$ of initial values.

The D- Penicillamine therapy in hepatic WD of the present series caused gradual decrease in serum bilirubin from initial value of 10.1 $( \pm 9.64) \mathrm{mg} / \mathrm{dl}$ to a value of $3.2( \pm 3.36) \mathrm{mg} / \mathrm{dl}$ at final follow up. Similarly SGPT decreased from admission value of $80.56( \pm 73.86) \mathrm{IU} / \mathrm{L}$ to final follow up value of $39.60( \pm 39.66) \mathrm{IU} / \mathrm{L}$ and significant reduction in INR from admission value of $1.64( \pm 0.6) \mathrm{sec}$ to a final value of 1.2 $( \pm 0.45) \mathrm{sec}$. These findings are supported by Roberts and Schilsky $(2003)^{3}$, Durand et al $(2001)^{17}$, and Ala et al $(2007)^{1}$. Roberts and Schilsky (2003) described that improvement in synthetic function of liver and clinical sign like Jaundice occurs during the first 2 to 6 months of treatment, but further recovery can occur during the first year of treatment. Durand et al (2001) experienced that early administration of D-penicillamine in patients with severe hepatic insufficiency was associated with survival without liver transplantation. Ala et al $(2007)^{1}$ stated that high bilirubin, prolonged prothrombin time usually improved with Dpenicillamine treatment in patients of WD with severe liver disease.

In neurological WD clinical improvement was not so evident in early stage but definite improvement was noticed in biochemical parameters as reflected by progressive increase in excretion of urinary copper, increasing trend of improvement in disability score and other clinical parameters on follow up.

Following treatment with penicillamine, though the disability score increased gradually but it was not statistically significant. Similarly dystonia and muscle power improved but did not reach to the significant level. The non significant neurological improvement was probably related to the irreversible changes in basal ganglia as part of pathogenesis. These findings were supported by Kalra et al (2000) and Ala et al $(2007)^{1,2}$. In a study with 25 Indian children of WD treated with D-penicillamine, zinc, pyridoxine and low copper diet, Kalra et al found improvement in majority, residual dysarthria in seven, prolonged persistence of $\mathrm{K}-\mathrm{F}$ ring in 15, and complications like renal tubular acidosis with osteopenia in one. Ala et al (2007) described that patients with neurological WD showed gradual clinical and cerebral MRI improvement with penicillamine treatment.

One of our hepatic WD patients also had joint pain, difficulty in walking. X-ray of wrist and ankle joints showed widening of epiphysis of both wrist and ankle joints. After treatment with dicaltrol, calcium and penicillamine, joints became normal in final x-ray follow-up. This observation was supported by Roberts and Schilsky (2003) ${ }^{3}$. They reported that patients with WD may present with important extrahepatic manifestation like premature osteoporosis and arthritis.

Side effects:

One of our patients developed proteinuria as a side effect of penicillamine in which it was replaced by zinc. Another two hepatic WD developed neurological symptoms like dystonia, dysarthria while on treatment. All these recognized side effects are supported by Roberts $\&$ Schilsky ${ }^{3}$. Roberts and Schilsky stated that penicillamine is associated with numerous side effects. Early sensitivity reaction includes fever, cutaneous eruption, neutropenia, thrombocytopenia and proteinuria and late 
reaction include nephrotoxicity heralded by proteinuria or the appearance of other cellular elements in the urine for which discontinuation of penicillamine should be immediate.

\section{Final outcome:}

Out of 16 cases, 7 improved of which 4 in hepatic and 3 in neurological group, 3 hepatic WD expired and 2 developed neurological manifestations. In neurological WD 4 cases remained unchanged till study period though 24 hours UC excretion was significant in all cases. The improvement in majority of the cases treated with D-penicillamine had also been reported by Kalra et al, Shakya et al and Durand et al 2,14,17.

\section{Conclusion:}

About half of patients with WD treated by Dpenicillamine were improved. Adequate cupriuresis occurred at three months; none achieved maintenance cupriuresis till one year after administration of the drug. All the symptoms and biochemical markers of WD improved gradually. No significant side effect was observed in the majority.

\section{References:}

1. Ala A, Walker AP, Ashkan K, Dooley JS, Schilsky ML. Wilson's disease. Lancet 2007; 369:397-408.

2. Kalra V, Khurana D, Mittal R. Wilson's diseaseEarly onset and lessons from a pediatric cohort in India. Indian Pediatr. 2000; 37: 595-601.

3. Roberts EA, Schilsky ML. AASLD practice guidelines: a practice guideline on Wilson disease. Hepatology. 2003; 37: 1475-92.

4. Das S K, Ray K. Wilson's disease: an update. Nat Clin Prac Neurol. 2006; 2: 482-93.
5. Pandit A, Bavdekar A and Bhave S.Wilson's disease. Indian J Pediatr. 2002: 69: 785-91.

6. Roberts EA, Schilsky ML; AASLD Practice guideline: diagnosis and treatment of Wilson Disease: an update. Hepatology. 2008; 47: 2089-2111.

7. Shen L, Ji HF. Adjunctive vitamin E treatment in Wilson disease and suggestions for future trials. Hepatology 2010; 51:1864-5.

8. Beheshti M, Ghotbi Sh. Wilson's Disease: a brief review. Shiraz-E- Med J. 2008; 9: 35-41.

9. Wilson Disease. In: Kalra V. eds. Practical Paediatric Neurology. 1st ed. New Delhi: Arya Publications; 2002: p. 172-4.

10. Diseases with several types of movement disorders.In: Farnandez E, Alvarez Aicardi J. eds, Movement Disorders in Children. 1st ed. London: Mackeith Press; 2001: p. 13, 130-41.

11. Motor system-General, Reflexes In: Fuller G. Neurological examination made easy. 3rd ed. Edinburgh: Churchill Livingstone; 2004: p. 119, 147.

12. Mahony FI, Barthel D. Functional evaluation: The Barthel Index. Maryland State Med J. 1965; 14: 56-61.

13. Yuce A, Kocak N, Demir H, et al. Evaluation of diagnostic parameters of Wilson's disease in childhood. Indian J Gastroenterol. 2003; 22: 1398-1401.

14. Shakya S, Agarwal JP. Clinical profile of Wilson's disease. Nepal J Neurosci. 2004; 1: 120-2.

15. Karim MB, Rahman MM, Islam MS. Wilson's Disease with Hepatic Presentationin Childhood. Mymensingh Med J. 2007; 17: 29-31.

16. Bertrand MVC, Degenhart HJ, Visser HKA, Sinaasappel M, Bouquet J. Oral zinc sulphate for Wilson's disease. Arch Dis Child. 1985; 60: 656-9.

17. Durand F, Bernuau J, Giostra E et al. Wilson's disease with severe hepatic insufficiency: beneficial effects of early administration of Dpenicillamine. Gut. 2001; 48: 849-52. 